\title{
Epidemiology of Hodgkin's disease
}

\author{
HARVEY ROTHMAN, DO \\ NATHAN FREED, DO
}

Hodgkin's disease, although a relatively uncommon malignancy, is one that frequently affects adolescents and young adults. The cure rate is very high; however, treatment for advanced-stage disease with chemotherapy or radiation therapy (or both) can lead to significant immediate and long-term morbidity, and, while rare, increased mortality from the development of secondary malignancies. There have been numerous epidemiologic reports citing a number of factors associated with the increased risk for the development of Hodgkin's disease. Many of these factors are related to environmental, socioeconomic, and genetic conditions. This report describes the development of Hodgkin's disease in two brothers and discusses factors associated with the increased risk of development of Hodgkin's disease, particularly in these cases.

Hodgkin's disease is an uncommon malignancy making up $1.3 \%$ of all newly diagnosed cancers in the United States. Only 6900 cases were reported in $1986 .{ }^{1}$ The incidence, however, appears to be increasing each year. In addition, because the average age of patients at diagnosis is 32 years, the economic impact with respect to total person-years of life lost for all lymphomas ranks fourth among all cancers.

Hodgkin's disease is also of great interest, not only because of the high curability rate, but because of the speculation that this disorder has a contagion etiology, perhaps due to an infectious agent. The implications, of course, are substantial. This report will discuss the epidemiology of Hodgkin's disease based on the cases of two brothers who had this lymphoma diagnosed within 1 year of each other.

\section{Report of cases}

In July 1982, Joseph, a 19-year-old man, had Hodgkin's disease, mixed-cellularity type, diagnosed after he had undergone a right cervical lymph node biopsy. Symp- toms had begun 3 months before diagnosis with swelling of the left, then the right side of his neck with associated fevers (temperature $>101^{\circ} \mathrm{F}$ ), night sweats and an approximate 8- to 16 -lb weight loss. The disease was staged as stage IIB according to the Ann Arbor classification after bilateral cervical, bilateral supraclavicular, and bilateral axillary lymphadenopathy was found on physical examination.

A chest film and a computed tomography scan revealed an anterior and middle mediastinal mass (greater than a third of the intrathoracic diameter). Findings of liverspleen scan, bipedal lymphangiogram, and gallium 57 scan (below the diaphragm), were normal, and results of bilateral bone marrow biopsies were negative. The patient achieved a complete clinical remission in February 1983 after undergoing six cycles of a chemotherapy regimen of nitrogen mustard, vincristine, procarbazine, and prednisone (MOPP) combined with radiation therapy (3600 rad) administered by "sandwich" technique. $\mathrm{He}$ maintained his complete remission until October 1987 , at which time his disease relapsed.

In June 1983, Joseph's 18-year-old brother had Hodgkin's disease, nodular sclerosing type, diagnosed after undergoing a right supraclavicular lymph node biopsy. His symptoms had begun 3 months earlier with vague, nondescript, retrosternal chest discomfort. During the interim, he experienced fevers with temperatures $>101^{\circ} \mathrm{F}$ and night sweats, but had no significant weight loss $(>10 \%)$. At the time of diagnosis, he had increasing retrosternal chest discomfort. A chest x-ray film was obtained and revealed a widening of the mediastinum. His disease was staged as stage IIB after physical examination revealed right-sided supraclavicular and bilateral axillary lymphadenopathy, in addition to the chest radiographic findings. Computerized tomography scan of the abdomen and pelvis and gallium 57 scan (below the diaphragm) showed no abnormalities, and results of bilateral bone marrow biopsies were negative. The patient achieved a complete clinical remission in February 1984 after undergoing a similar combined chemotherapy (MOPP, six cycles) and radiation therapy (4000 rad) sandwich-technique regimen. He has maintained complete remission until the present.

Both brothers lived with four other siblings (total five brothers and one sister). Joseph has an identical twin, William. The order of birth (by age, from oldest to youngest) is: William, Joseph, John, James, Lori, and Kenney. There is no other family history of any type of lymphoma. Both patients had no history of tonsillectomy, 
appendectomy, infectious mononucleosis, prior malignancy, immunosuppresive therapy, or other medication use (such as phenytoin or amphetamines). Both brothers are Catholic and grew up in a lower middle-class, single-family home with a total of seven or eight members (some family members were occasionally absent). Joseph had a very small circle of playmates while growing up whereas James had a large number of playmates. Both attended rather small parochial elementary and high schools, and both completed (and had their disease diagnosed while attending) community colleges. Hodgkin's disease developed in two elementary school classmates of James within the past year while they were attending college. Both of our patients' parents attended school only to the high school level.

\section{Discussion}

As stated, Hodgkin's disease is an uncommon malignancy. However, since 1832, when Thomas Hodgkin first described seven fatal cases of the disease that now bears his name, there have been frequent reports of clusters of cases, more so than would be otherwise expected, leading to speculation of the role of a "contagion" in the etiology. The two cases we described here may give credence to this speculation.

In 1982, Haim and associates ${ }^{2}$ reported a statistically significant increased incidence of Hodgkin's disease in first-degree blood relatives. Four pairs of immediate relatives with Hodgkin's disease were found among 183 patients in whom the lymphoma was diagnosed over a 20 -year period. The expected number during that period was 0.45 . Consequently, close relatives of patients with Hodgkin's disease had a ninefold increased risk of the disease. In 1983, Kerzin-Storrar and coworkers ${ }^{3}$ reported a similar fourfold increase (six cases observed versus 1.4 expected). Furthermore, Grufferman and associates ${ }^{4}$ reported on an incidence survey of Hodgkin's disease in Greater Boston over a 14-year-period where the expected number was 0.7 . The survey discovered five sibling pairs, younger than 45 years, thus showing a sevenfold increased risk in this group. Additionally, among 13 pairs of siblings, they identified 12 who were sex concordant, with an expected number of 6.8 . The number of these cases plus similar cases in a literature review ${ }^{4}$ suggests that siblings of the same sex as an affected person have a risk of the development of Hodgkin's disease double that of siblings of the opposite sex. Therefore, the role of genetic susceptibility in the development of Hodgkin's disease must be considered; however, due to the lack of an identified pattern of inheritance, little is known.
In 1983 , Berberich and associates ${ }^{5}$ reported the use of human leukocyte antigen (HLA) technique in studying familial cases of Hodgkin's disease. They found that the haplotype concordance among patients was greater than expected by chance alone, thereby providing a new method for assessing linkage of HLA and disease susceptibility, and new evidence for the genetic control susceptibility to Hodgkin's disease. Such susceptibility could possibly contribute to the unlikely event of Hodgkin's disease developing in two brothers. Interestingly, to date, Hodgkin's disease has not developed in Joseph's identical twin, William.

The number of siblings is thought to have an inverse relationship to the development of Hodgkin's disease, with the risk among patients with five or more siblings nearly half that of patients with none or one sibling. ${ }^{6}$ The number of our patients' siblings does not fit this trend. In addition, Gutensohn and $\mathrm{Cole}^{6}$ reported that the risk of Hodgkin's disease is reduced among patients of late birth order. This association apparently holds true for Joseph with his early birth order and increased risk of subsequent later development of Hodgkin's disease and also for Lori and Kenney with their later birth order and decreased risk of Hodgkin's disease, which to date has not developed.

Our patients and their family lived in a singlefamily housing unit. Gutensohn and $\mathrm{Cole}^{6}$ also reported that the risk of Hodgkin's disease in persons who lived in multiple-family dwellings was half that of persons in single-family homes. Perhaps this is an additional reason for both brothers having Hodgkin's disease.

Joseph had many playmates while growing up as a child, whereas James had fewer. It has been reported that the number of neighborhood playmates a child has at age 8 is inversely proportional to the risk of the development of Hodgkin's disease. ${ }^{7}$ The brothers' parents both continued their education only through high school, and their socioeconomic class could be classified as lower middle. Both of these variables are associated with increased risk of Hodgkin's disease in their offspring. ${ }^{7}$ The risk of Hodgkin's disease is twice as great in Jews as Catholics, ${ }^{6}$ but as already noted, our patients' family is Catholic.

The issue has been debated regarding the risk of Hodgkin's disease in persons who have undergone prior tonsillectomy. 8,9 The general consensus appears to be that tonsillectomy is not a cause nor does it increase the risk of Hodgkin's disease. If it is a cause or increased risk factor, the effect is 
complex and modified by factors related to family size. Neither brother had had a tonsillectomy. Persons with Hodgkin's disease have been found to have a higher incidence of prior appendectomy ${ }^{10}$; however, neither brother had had an appendectomy.

Long-term use of certain medications such as phenytoin has been associated with the development of lymphoma, especially Hodgkin's disease. ${ }^{11}$ Neither brother had been taking phenytoin or any other medication on a long-term basis.

There is some evidence to suggest that chronic exposure to wood or wood dust may increase the risk of Hodgkin's disease. Milham and Hesser ${ }^{12}$ analyzed the death certificates of 1594 white males who had died of Hodgkin's disease compared with those of matched controls and calculated the risk factor of 2.3 for those who were woodworkers. Longterm exposure to wood dust may be part of a casual pathway of providing a continuing antigenic stimulation. Neither brother had such exposure.

There has been much speculation as to an association between an infectious agent and the development of Hodgkin's disease based on early epidemiologic studies of close contacts among individuals and the later development of Hodgkin's disease. Initial reports ${ }^{13-15}$ of clusters of Hodgkin's disease in schools and classrooms exceeding the expected rates of development of the disease sparked the concern of a viral contagion as an etiologic agent. Because Hodgkin's disease predominately affects adolescents and young adults, the Epstein-Barr virus, the causative agent of infectious mononucleosis, has been implicated. The debate continues.

Vianna and associates ${ }^{13,14}$ and Zack and associates $^{15}$ suggested evidence of transmission of Hodgkin's disease in schools, with an increased risk of development among classmates. Grufferman and associates ${ }^{16}$ and Smith and coworkers, ${ }^{17}$ however, showed no excess development of Hodgkin's disease between classmate pairs that might have occurred otherwise by chance. Joseph and James both attended the same elementary and high schools, with 2 years intervening their entry. In addition, two elementary school classmates of James have since had Hodgkin's disease develop while in college.

A report of clusters of cases of Hodgkin's disease in school and military cohorts ${ }^{18-21}$ led to the suggestion of involvement of an infective agent in the etiology. The Epstein-Barr virus has been implicated for two main reasons: (1) There is an increased incidence of lymphomas developing after infectious mononucleosis compared with control subjects who have not had this disease; and (2) patients with Hodgkin's disease more so than control subjects without this lymphoma have elevated antibody titers against Epstein-Barr virus. No true virus, however, has been found in tumor specimens from patients with Hodgkin's disease. Neither brother had a history of infectious mononucleosis.

The increased incidence of malignant disorders has been observed in renal allograft transplant recipients. The most frequently occurring malignancies are cancer of the skin and lip (35\% of cases), followed by lymphoma ( $27 \%){ }^{22}$ The most common lymphoma is large-cell non-Hodgkin's lymphoma (NHL), followed by Kaposi's sarcoma. ${ }^{22}$ Hodgkin's disease infrequently is associated as a tumor developing subsequent to transplant procedures. It was thought that the association between transplant recipients and the development of cancer is related to the immune suppression brought on by immunosuppressive medication, (ie, azathioprine, cyclophosphamide, or prednisone) required by the patient after transplantation. ${ }^{22}$ There is no evidence of preexisting neoplasms in either the donors or the recipients prior to transplantation. Lymphoreticular tumors are also known to occur with increased frequency among patients with primary immunodeficiency disorders. ${ }^{22}$

A similar relationship probably exists between patients with the acquired immune deficiency syndrome (AIDS) and their high risk for malignancies, namely, Kaposi's sarcoma and NHL (Burkitt's lymphoma, Burkitt's-like lymphoma-diffuse undifferentiated NHL, and immunoblastic sarcoma). ${ }^{23}$ Again, Hodgkin's disease is an infrequent occurrence.

Neither patient had a history of primary immunodeficiency disorder nor a human immunodeficiency virus (HIV) infection or exposure.

\section{Summary}

It appears two brothers had Hodgkin's disease develop 9 months apart. Certain risk factors or associations with the increased risk for Hodgkin's disease were noted, including first-degree blood relatives, sibling-pairs, sex-concordance, early birth order (Joseph), single-family housing unit, small cohort of childhood playmates (James), lower level of parent's educational attainment, middle-class status, and increased clustering of cases in school populations.

Our paper notes the factors associated with the risk for Hodgkin's disease, a malignancy which, 
though rare, and fortunately highly curable, still has great impact in a young population.

\section{The authors wish to acknowledge the assistance of Nena Pappas and Florence Barracliffe for their as- sistance in the preparation of this paper.}

1. Silverberg E, and Lubera J: Cancer statistics, 1986. CA 1986;36:9-25. 2. Haim N, Cohen Y, Robinson E: Malignant lymphoma in first-degree blood relatives. Cancer 1982;49:2197-2200.

3. Kerzin-Storrar L, Faed MJW, MacGillivray JB, et al: Incidence of familial Hodgkin's disease. $B r$ J Cancer 1983;47:707-712.

4. Grufferman S, Cole P, Smith PG, et al: Hodgkin's disease in siblings. $N$ Engl J Med 1977;296:248-250.

5. Berberich FR, Berberich MS, King MC, et al: Hodgkin's disease susceptibility: Linkage to HLA locus demonstrated by a new concordance method. Hum Immuno 1983;6:207-217.

6. Gutensohn N, Cole P: Childhood social environment and Hodgkin's disease. N Engl J Med 1981;304:135-140.

7. Gutensohn N, Shapiro DS: Social class risk factors among children with Hodgkin's disease. Int $J$ Cancer 1982;30:433-435.

8. Vianna NJ, Greenwald P, Davies JNP: Tonsillectomy and Hodgkin's disease: The lymphoid tissue barrier. Lancet 1971;1:431-432.

9. Newell GR, Rawlings W, Kannear BK, et al: Case-control study of Hodgkin's disease. I. Results of the interview questionnaire. J Natl Cancer Inst 1973;51:1437-1440.

10. Bierman HR: Human appendix and neoplasia. Cancer 1968;21:109118.

11. Hyman GA, Sommers SC: The development of Hodgkin's disease and lymphoma during anticonvulsant therapy. Blood 1966;28:416-427.
12. Milham S, Hesser JE: Hodgkin's disease in woodworkers. Lancet 1967;1:136-137.

13. Vianna NJ, Greenwald P, Davies JNP: Extended epidemic of Hodgkin's disease in high school students. Lancet 1971;1:1209-1210.

14. Vianna NJ, Polan AK: Epidemiologic evidence for the transmission of Hodgkin's disease. $N$ Engl J Med 1973;289:499-502.

15. Zack NM, Heath CW, Andrews MD, et al: High school contact among patients with leukemia and lymphomas. $J$ Natl Cancer Inst 1977;59:1343. 1349.

16. Grufferman S, Cole P, Levitan TR: Evidence against transmission of Hodgkin's disease in high schools. N Engl J Med 1979;300:1006-1011.

17. Smith PG, Pike MC, Kinlen LJ, et al: Contacts between young patients with Hodgkin's disease. Lancet 1977;2:59-62.

18. Rosdahl N, Larsen SO, Clemmesen J: Hodgkin's disease in patients with previous infectious mononucleosis: 30 years' experience. $\mathrm{Br} \mathrm{Med}$ $J$ 1974;2:253-256.

19. Connelly RR, Christine BW: A cohort study of cancer following infectious mononucleosis. Can Res 1974;34:1172-1178.

20. Carter CD, Brown TM, Herbert JT, et al: Cancer incidence following infectious mononucleosis. Am J Epidemiol 1977;105:30-36.

21. Kvale G, Hoiby EA, Pedersen E: Hodgkin's disease in patients with previous infectious mononucleosis. Int $J$ Cancer 1979;23:593-597.

22. Penn I: The incidence of malignancies in transplant recipients. Trans. plant Proc 1975;7:323-326.

23. Allen JR: Epidemiology of the acquired immunodeficiency syndrome (AIDS) in the United States. Semin Oncol 1984;11:4-11.

From the department of medicine, division of hematology/ oncology, University of Medicine and Dentistry of New Jersey School of Osteopathic Medicine, Stratford, NJ.

Reprint requests to Dr Rothman, $301 \mathrm{~S}$ Central Plaza, Stratford, NJ 08084-1504. 
Evidence directly linking elevated cholesterol and CAD made you take a closer look at lipid levels

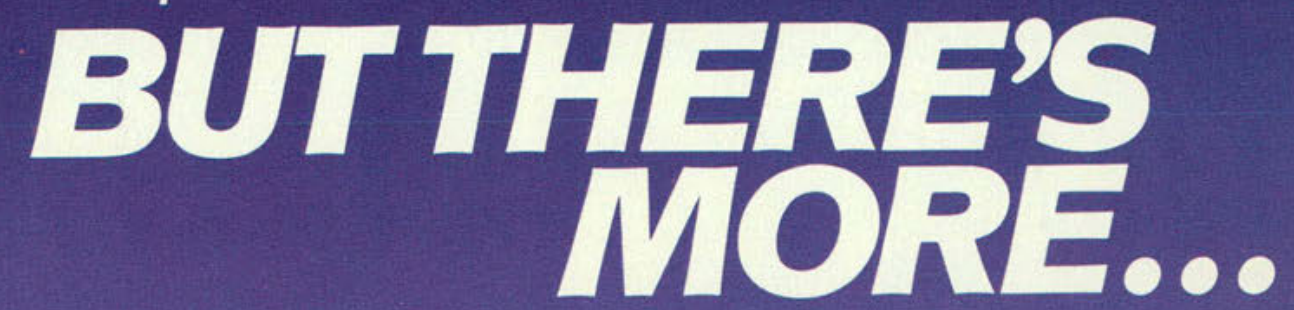


Lorelco is indicated for the reduction of elevated serum cholesterol in patients with primary hypercholesterolemia, as an adjunct to diet.
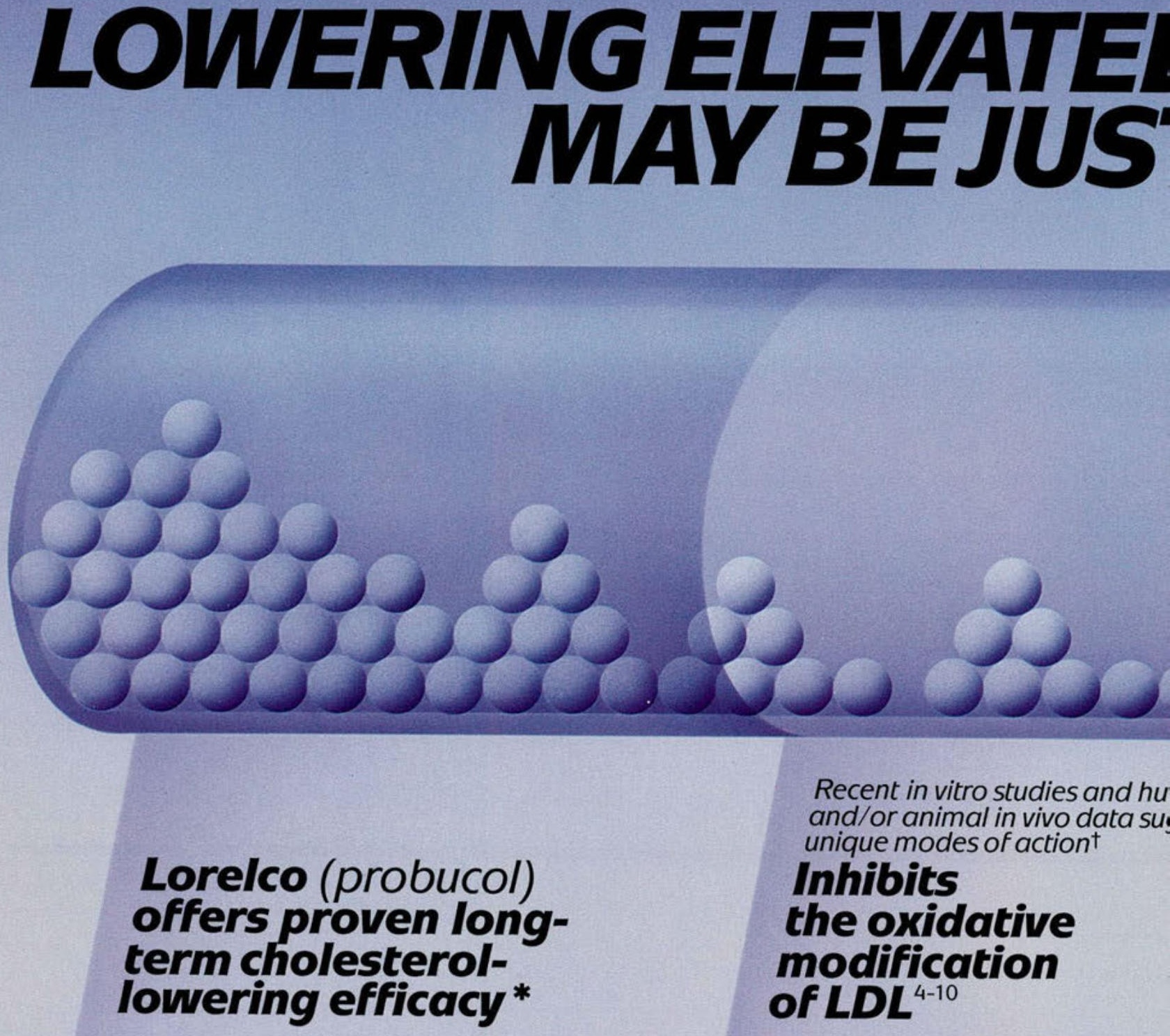

- Lowers total cholesterol by up to $27 \%^{1.2}$

- Maintains lower levels as proven over a 12-year period ${ }^{3}$

- Increases fractional rate of LDL catabolism

(See Clinical Pharmacology)

*The effect of probucol-induced reduction of serum cholesterol or triglyceride levels or reduction of HDL-cholesterol levels on morbidity or mortality due to coronary heart disease has not been established.

References: 1.SAfr Med J1982;62:7-11. 2. Arch Intern Med 1981:141:1428-1432. 3. Data on file, MERRELL DOW PHARMACEUTICALS INC., Cincinnati, Ohio 45242 9553. 4. Am J Cardiol 1988.62:318-368.5. Acherosclerosis 1986:62.209-217. 6. Proc Natl Acad Sci USA 1987:84:5928-5931.7. Clin Invest 1986: 62:209-217. 6. ProcNati Acad Sci USA 1987:84.5928-5931. 7.J Cin Invest 1986:77:641-644. 8. Proc Natl Acad Sci USA 1987;84:7725-7729. 9. Am J Cardiol 1988;62:68-12B 10. Am J Cardiol 1986:57:16H-21H. 11. Am J Cardiol 1988:62:57B-59B. 12. Am Cardiol 1988:62:66B-72B. 13. Am J Cardiol 1988;62:73B-76B. 14. Am J Cardiol 1971:136:1216-1221.17. Jpn JPharmacol 1986:41:211-222 18. Am J Cardiol 1986:57:29H-35H

Recent in vitro studies and hu and/or animal in vivo data su. unique modes of action ${ }^{\dagger}$

\section{Inhibits the oxidative modification of $\mathbf{L D L}^{4-10}$}

- Modified (cytotoxic) LDL may play a role in foam cell formation ${ }^{4-10}$

-foam cell formation is an initial step in atherosclerotic plaque development $t^{4-10}$ - Inhibits development of atherosclerotic lesions in animals $\$ 6,8$

†Data are only used to explain the unique modes of action of Lorelco; no clinical benefit is implied.

Lorelco is not an innocuous drug and strict attention should be paid to the Indications, Contraindications, Warnings, and Precautions sections of Prescribing Information. 


\section{EVELS HE BEGINNING}

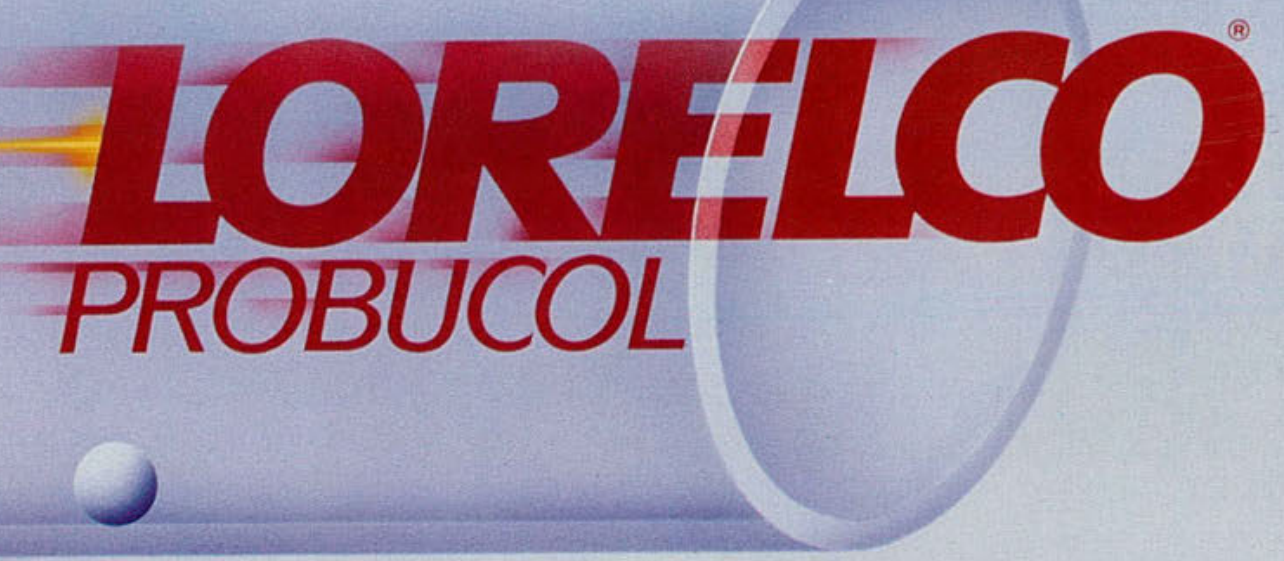

Recent in vitro studies and human and/or animal in vivo data suggest unique modes of action ${ }^{\dagger}$

\section{Enhances}

HDL-mediated reverse cholesterol transport $\$ 3,11-14$

- Lorelco has been shown to regress atherosclerotic lesions in animals $\$ 15-17$

- The effects of probucol on HDL-cholesterol may reflect improved dynamics of HDLmediated reverse cholesterol transport $1 / 3,12,14,18$

$\ddagger$ See Contraindications, Warnings, and Animal Pharmacology and Toxicology.

$\S$ These effects of probucol on $L D L$ (increase in catabolic rate) and HDL-cholesterol may be linked to the observed increased excretion of fecal bile acids, a final metabolic pathway for elimination of cholesterol from the body. (See Clinical Pharmacology.)

See Prescribing Information appearing on next page.

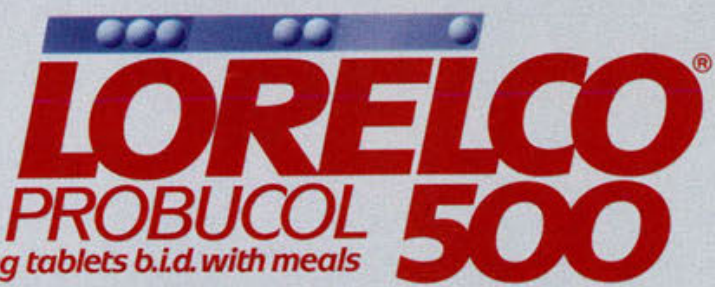

$500 \mathrm{mg}$ tablets b.i.d. with meals

TAKES YOU TO THE NEXT LEVEL OF CHOLESTEROL CONTROL

//The probable benefits obtained from $L D L-$ cholesterol reduction must be weighed against the possible risk of a reduction in HDL-cholesterol when assessing the response of patients to probucol. If satisfactory lipid alteration is not achieved, the drug should be discontinued.

(See Precautions.) 


\section{Lorelco ${ }^{\circledast}$ Tablets (probucol)}

DESCRIPTION: Lorelco (probucol) film-coated tablets for oral administration contain $250 \mathrm{mg}$ or $500 \mathrm{mg}$ of probucol per hydroxypropyl methylcellulose 2910 , iron oxide, lactose, magnesium stearate, microcrystalline cellulose, polysorbate 80 , talc, and titanium dioxide. Lorelco is an agent for the reduction of elevated serum cholesternt. ethylidene) bis(thio) bis [2,6-bis(1,1-dimethyl-
ethyl)phenol] . Its chemical structure does not resemble that of any other available cho-
lesterol-lowering agent. It is lipophilic.

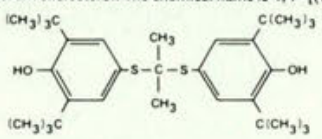

CLINICAL PHARMACOLOGY: Lorelco lowers total serum cholesterol and has relatively little effect on serum triglycerides Patients responding to probucol exhibit a decrease in low-density lipoprotein (LDL) cholesterol. Cholesterol is reduced not only
in the LDL fraction, but also in the high-density lipoprotein (HDL) fraction with proportionately greater effect on the high-density portion. Epidemiologic studies have shown that both low $\mathrm{HDL}$-cholesterol and high LDL-cholesterol are independent risk tactors or no effect reported on very low-density lipoprotein (VLDL).

Studies on the mode of action of Lorelco indicate that it increases the fractional rate of LDL catabolism. This effect may be linked to the observed increased excretion of fecal bile acids, a final metabolic pathway for the elimination of cholesterol from the body.
Lorelco also exhibits inhibition of early stages of cholesterol biosynthesis and slight inhibition of absorption of dietary cholesterol. There is no increase in the cyclic precursors of cholesterol, namely desmosterol and 7-dehydrocholesterol. On this Absorption of Lorelco from the gastrointestinal tract is limited and variable. When it is administered with food, peak blood levels are higher and less variable. With continuous administration in a dosage of $500 \mathrm{mg}$ b.i.d., the blood levels of an individual
gradually increase over the first three to four months and thereatter remain fairly constant. in 116 patients treated with Lorelco
for periods of three months to one year, the mean blood level was $23.6 \pm 17.2 \mathrm{mcg} / \mathrm{mL}( \pm S$. D. Franging to $78.3 \mathrm{mco} / \mathrm{mL}$. for periods of three months to one year, the mean blood level was $23.6 \pm 17.2 \mathrm{mcg} / \mathrm{mL}( \pm S .0$.) ranging to $78.3 \mathrm{mcg} / \mathrm{mL}$.
Levels observed after seven years of treatment in 40 patients yielded an average value of $21.5 \pm 16.5 \mathrm{mcg} / \mathrm{mL}( \pm S .0$.) ranging to $62.0 \mathrm{mcg} / \mathrm{mL}$. In a separate study in eight patients, blood levels averaged $19.0 \mathrm{mcg} / \mathrm{mL}$ at the end of 12 months of tir

In December 1984, a National Institutes of Health Consensus Development Conference Panel1 concluded that lowering definitely In December 1984, a National Institutes of Health Consensus Development Conference Panell concluded that lowering definitely
elevated blood chiolesterol levels (specifically blood levels of LOL-cholesterol) will reduce the risk of heart attacks due to coronary heart disease. The effect of probucol-induced reduction of serum cholesterol or triglyceride levels, or reduction of

INDICATIONS AND USAGE: Serious animal toxicity has been encountered with probucol. See WARNINGS and ANIMAL
PHARMACOLOGY AND TOXICOLOGY sections. Probucol is not an innocuous drug and strict attention should be paid to the INDICATION

Drug therapy should not be used for the routine treatment of elevated blood lipids for the prevention of coronary heart disease. Dietary therapy specific for the type of hyperlipidemia is the initial treatment of choice. Excess body weight may be an important
factor and should be addressed prior to any drug therapy. Physical exercise can be an important ancillary measure. Contributory
disease such as hypothyroidism or diabetes mellitus should be looked for and adequat disease such as hypothyroidism or diabetes mellitus should be looked for and adequately treated. The use of drugs should be considered only when reasonable attempts have been made to obtain satistactory results with nondrug methods. If the decision
ultimately is to use drugs, the patient should be instructed that this does not reduce the importance of adhering to diet. The selection of patients for cholesterol-lowering drug therapy should take into account other important coronary risk factors such as smoking, hypertension, and diabetes mellitus. Consideration should be given to the efficacy, salety, and compliance
factors for each of the cholesterol-lowering drugs prior to selecting the one most appropriate for an individual patient. Lorelco may be indicated for the reduction of elevated serum cholesterol in patients with primary hypercholesterolemia (Types
llia and Ilb hyperlipoproteinemia). ${ }^{2}$ whose elevated LDL-cholesterol has not responded adequately to diet, weight reduction, and control of diabetes mellitus. Lorelco may be useful to lower elevated LDL-cholesterol that occurs in those patients, with
combined hypercholesterolemia and hypertriglyceridemia (Type IIb) due to elevation of both LDL and VLDL, but it is not
indicated where hypertriglyceridemi a combined hypercholesterolemia and hypertriglyceridemia (Type IIb) due to elevation of both LDL and VLDL, but it is nol cholesteroi represents a primary lipid disorder, it should be determined that patients being considered for treatment with Lorelco have an elevated LDL-cholesterol as the cause for an elevated total serum cholesterol. This may be particularly relevant for
patients with elevated triglycerides or with markedly elevated $\mathrm{HDL}$-cholesterol values, where non-DL fractions may contribute
significantly to total cholesterol levels without apparent increase in cardiovascular risk. In most patients, LDL-cholesterol may significantly to total cholesterol levels without app
be estimated according to the following equation:

$$
\text { LDL-cholesterol }=\text { Total cholesterol }-[(0.16 \times \text { triglycerides })+\text { HDL-cholesterol }]
$$

When total triglycerides are greater than $400 \mathrm{mg} / \mathrm{dL}$, this equation is less accurate. In such patients, LDL-cholesterol may be It is not always possible to predict from the lipoprotein type or other factors which patients will exhibit favorable results. Lipid The effect of probucol-induced reduction of serum cholesterol or triglyceride levels, or reduction of HDL-cholesterol levels on morbidity or mortality due to coronz

CONTRAINDICATIONS: (See also WARNINGS and PRECAUTIONS.) Lorelco is contraindicated in patients who are known to have a hypersensitvity to it. Lorelco is contraindicated in patients with evidence of recent or progressive myocardial damage or
findings suggestive of serious ventricular arthythmias or with unexplained syncope or syncope of cardiovascular origin. Lorelco is contraindicated in patients with an abnormally long QT interval.

WARNINGS: SERIOUS ANIMAL TOXICITY HAS BEEN ENCOUNTERED WITH PROBUCOL IN RHESUS MONKEYS FED AN Prolongation of the QT interval can occur in patients on Loreico. Serious arrhythmias have been seen in and a concomitant antiarrhythmic drug. The following precautions are deemed prudent: 1. Patients should be advised to adhere to a low cholesterol, low fat diet at the start of treatment with Lorelco and throughout the 2. An ECG should be done prior to starting treatment and repeated at appropriate intervals during treatment. If an abnormally long QT interval is observed, the possible benefits and risks should be carefully considered before making a decision to
continue Lorelco. Lorelco therapy should be discontinued or not started if the OT interval at an observed heart rate on a resting ECG is
persistently more than one of the values listed below.

\begin{tabular}{ccc}
\multirow{2}{*}{$\begin{array}{c}\text { Observed Heart Rate } \\
\text { (beats/min) }\end{array}$} & \multicolumn{2}{c}{$\begin{array}{c}\text { OT Interval in sec (15\% above the } \\
\text { upper limit of normal) }\end{array}$} \\
\cline { 2 - 3 } & Males & Females \\
\hline 40 & 0.56 & 0.58 \\
50 & 0.52 & 0.53 \\
70 & 0.49 & 0.50 \\
80 & 0.45 & 0.47 \\
86 & 0.43 & 0.44 \\
92 & 0.42 & 0.43 \\
100 & 0.40 & 0.40 \\
109 & 0.39 & 0.38 \\
120 & 0.37 & 0.36 \\
133 & 0.36 & 0.35
\end{tabular}

"Values calculated from Burch GE. Winsor T. A primer of electrocardiography. Philadelphia, PA: Lea and Febiger, 1958; D.
272 (Table 6).

3. Patients developing unexplained syncope or syncope of cardiovascular origin should have Lorelco therapy discontinued and
should have $E C G$ surveillance.

. Orugs that prolong the OT interva

a. An increase in the dose of the drue more inkely to be associated with ventricular tachycardia after:

. Addition of a second drug that prolongs the QT interval (including tricyclic antidepressants, class I and III antiarrhythmics, c. Hypokalemia or hypomagnesemia

d. Severe bradycardla due to intrinsic heart disease or drug effects on the atrial rate (beta-blockers) or AV block (digoxin). The use of Lorelco in patients receiving any of these drugs should be based on the conclusion that alternate methods of hypocholesterolemic therapy are either ineffective or not tolerated, and the poten The following conditons should be resolved or cor

The following conditions should be resolved or corrected prior to initiation of therapy with Lorelco:

a. Hypokalemia

b. Hypomagnesemia
c. Severe bradycardia due to intrinsic heart disease or drug effects on the atrial rate (beta-blockers) or AV block (digoxin).
d. Recent or acute myocardial infarction, ischemia, or inflammation. PRECAUTIONS General: Before instituting therapy with Lorelco, adequate baseline studies should be performed to determine that the patient
has persistently elevated total and LDL-cholesterol levels representing a primary hipid disorder, and that the increased
cholesterol is not due to secondary conditions such as hypothyroidism, cholesterol is not due to secondary conditions such as hypothyroidism, poorly controlled diabetes mellitus, obstructive liver
disease, nephrotic syndrome, or dysproteinemias. Serum lipld levels, including HDL-cholesterol, should be determined after an
overnight tast before treatment.

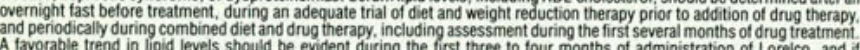
A favorable trend in lipid levels should be evident during the first three to tour months of administration of Lorelecto, and if
satistactory lipid alteration is not achieved, the drug should be discontinued. Lorelco lowers serum total and LDL-cholesterol, satistactory lipid alteration is not achieved, the drug should be discontinued. Lorelco lowers serum total and LDL-cholesterol. and also lowers HDL-cholesterol in most patients with elevated LDL-cholesterol. Epidemiologic studies within hyper-
cholesterolemic populations have shown that serum HDL-cholesterol is an independent, inversely correlated, risk factor for
coronary heart disease (see CLINICAL PHARMACOLOGY) Human studies which will attempt to confirm or deny the hypothesis coronary heart disease (see CLINICAL PHARMACOLOGY). Human studies which will attempt to confirm or deny the hypothesis
that drug-induced alteration in HDL-cholesterol affects cardiovascular risk are currently under evaluation. It is not known
whether Lorelco-induced reduction of serum HDL-cholesterol will affect cardiovascular risk since no long-term, controlled whether Lorelco-induced reduction of serum HOL-cholesterol will affect cardiovascular risk since no long-term, controlled been performed. The probable benefits obtained from LDL-cholesterol reduction must be weighed against the possible risk of a
reduction in HOL-cholesterol when assessing the response of each patient receiving Lorelco treatment. If satistactory lipid
alteration is not achieved, the drug should be discontinued.
Information for Patients: The patient should be instructed to adhere to a prudent diet. Females should be cautioned
becoming pregnant for at least six months after discontinuing Lorelco and should not breast-feed their infants during

Laboratory Tests: The physician should schedule periodic blood lipid determinations and periodic ECGs. (See WARI Elevations of the serum transaminases (SGOT, SGPT), bilirubin, alkaline phosphatase, creatine phosphokinase, uric acid urea nitrogen, and blood glucose above the normal range were observed on one or more occasions in various patients
with Lorelco. Most often these were transient andior could have been related to the patient's clinical state or other $m$ therapy. Although the basis for the relationship between Loreico and these abnormalities is not firm, the possibility that patients treated with Lorelco than in the patients who received placebo. If abnormal laboratory tests persist or worsen, it
signs consistent with the abnormal laboratory tests develop, or if systemic manifestations occur, Lorelco should be

Drug Interactions: The addition of clofibrate to Lorelco is not recommended, since the lowering effect on mean serum of either $\mathrm{LL}$ or total choles
lowering of HDL-cholesterol.

Neither oral hypoglycemic agents nor oral anticoagulants alter the effect of Lorelco on serum cholesterol. The dosage

Monkeys fed a high fat, high cholesterol diet admixed with probucol exhibited serious toxicity. (See WARNINGS and AN
PHARMACOLOGY AND TOXCOLOGY sections) Prolongation of the PHARMACOLOGY AND TOXICOLOGY sections.) Prolongation of the QT interval can occur in patients on Lorelco and sn
arrmhythmias have been seen in association with an abnormally long QT interval in patients on Lorelco. The addition of as drug that prolongs the QT interval (including tricyclic antidepressants, class I and III a nnt
increase the risk of serious arrtyythmia. (See CONTRAINDICATIONS AND WARNIGS.)

Carcinogenesis, Mutagenesis, Impairment of Fertility

In chronic studies, of two years' duration in rats, no toxicity or carcinogenicity was observed. These res
the lack of any adverse effect on fertility and the negative findings in tests for mutagenic activity in rats.

Pregnancy

Teratogenic Effects
Pregnancy - Category B: Reproduction studies have been pertormed in rats and rabbits at doses up to 50 times the and well-controlled studies in pregnant women. Because animal reproduction studies are not always predictive of $h$ hom
response, this drug should be used during pregnancy only if clearly needed. Furthermore, if a patient wishes to be
pregnant, it is recommended that the drug be withdrawn and birth control procedures be used for at least six months bea persistence of the drug in the body for prolonged periods. (See CLINICAL. PHARMACOLOGY.)

Labor And Delivery: The effect of Lorelco on human labor and delivery is unknown.

Nursing Mothers: It is not known whether this drug is excreted in human milk, but it is likely, since such excretion ha Pediatric Use: Safety and effectiveness in children have not been established.

ADVERSE REACTIONS

diarritea or loose

Cardiovascular
prolongation of the OT interval on ECG, syncope, ventricular arritythmias (ventricular tachycardia, torsades de
ventricular fibrillation), sudden death

Neurologic

headachie, dizziness, paresthesia, insomnia, tinnitus, peripheral neuritis

Hematologic

hemoglobin and/or hematocrit, thrombocytopenia

rash, pruritus, ecchymosis, petechiae, hyperhidrosis, fetid sweat

Genitourinary
impotency, nocturia

Ophthaimic

conjunctivitis, tearing, blurred vision

Endocrine

enlargement of multinodular goiter

Other

diminished sense of taste and smell, anorexia, angioneurotic edema

DRUG ABUSE AND DEPENDENCE: No evidence of abuse potential has been associated with Lorelco, nor evidence of psychological or physical dependence in humans.

OVERDOSAGE: There is a single report of a 15-kg, three-year-old, male child who ingested $5 \mathrm{~g}$ of probucol. Emes:
induced by ipecac. The child remained well, apart from a brief episode of loose stools and flatulence. No specific infor induced by ipecac. The child remained well, apart trom a brief episode of loose stools and flatulence. No specific infoi
is avaliable on the treatment of overdosage with Lorelco and no specific antidote is available. Probucol is not diay Treatment is symptomatic and supportive. Probucol has shown no identifiable acute toxicity in mice and rats. In

DOSAGE AND ADMINISTRATION: For adult use only. The recommended and maximal dose is $1000 \mathrm{mg}$ daily HOW SUPPLIED: $250 \mathrm{mg}$ round, white, film-Coated tablets imprin
code number 51 or LORELCO 250 . Bottles of 120 (NDC 0068-0051-52)

$500 \mathrm{mg}$ capsule-shaped, white, film-coated tablets, marked LORELCO 500 . Bottles of 100 (NDC 0068-0053-61)

Keep well closed. Store in a dry place. Avoid excessive heat. Dispense in well-closed light-resistant containers with
resistant closure.

ANIMAL PHARMACOLOGY AND TOXICOLOGY: In thesus monkeys, administration of probucol in diets con unusually high amounts of cholesterol and saturated fat resulted in the dea.th of tour of eight animals after several
Premonitory syncope was frequently observed and was associated with a pronounced prolongation of the $Q T$ intervals Premonitory syncope was frequently observed and was associated with a pronounced prolongation of the QT intervals
$50 \%$ longer than that observed in untreated monkeys. Serum levels of probucol greater than $20 \mathrm{mcg} / \mathrm{mL}$ were gen

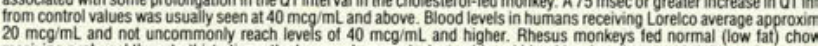
eceiving probucol three to thirty times the human dose equivalent achieved blood levels only one-third those of many $h$ subjects. No adverse effects were detected in these monkeys over an eight-year period of continuous drug administrat another study in rhesus monkeys, an atherogenic diet was fed for two years and dally treatment with probucol, separated
from the atherogenic meal, was carried out during the second year. Serum probucol levels ranged 20 to $50 \mathrm{mcg} m \mathrm{~mL}$ in five from the atherogenic meal, was carried out during the second year. Serum probucol levels ranged 20 to $50 \mathrm{mcg} / \mathrm{mL}$ in five
monkeys, and less in the remaining animals. Marked prolongation of the Qf interval in the ECG or syncopal behavior was
observed over the entire one-year treatment period Regression of observed over the entire one-year treatment period. Regression of gross a ortic lesions comparable to that observed in a
group of monkeys receiving cholestyramine was seen in animals receiving probucol. It should be emphasized that bot group of monkeys receiving cholestyramine was seen in animals receiving probucol. It should be emphasized that both
cholesterol and (DL-cholesterol were markedly reduced in this regression study. During the performance of a two-year $c$.

Subsequent experiments have indicated that probucol sensitizes the canine myocardium to epinephrine, resulting in ventr
fibrillation in many doss. Among the animal species in which probucol has ing
phenomenon of sudden death due to the sensitization of the myocardium to epinephrine. In contrast to findings in the phenomenon of sudden death due to the sensitization of the myocardium to epinephrine. In other studies, monkeys were given probucol either before and after, or only after myocardial infarction was induce
coronary artery ligation. In these studies, there was no difference between probucol- and placebo-treated groups with resp
either survival or detailed blind quantitation of myocardial changes (gross and histopathologic) Pror

Probucol has shown no identifiable toxicity in mice and rats. In these animals, the LDso (oral) is in
weight. In chronic studies of two years' duration in rats, no toxicity or carcinogenicity was observed. probucolias aninistereded orally is unabsorbed. For that which is absorbed, the biliary tract is the major pathway for clearance

Myocardial injury was produced in various groups of rats by one of the following procedures: aortic coarctation, cor
ligation, or cobalf or isoproterenol injection. Fifter probucol administration, no deleterious effects related to treatment as measured by survival and microscopic examination of myocardial damage.

Probucol was administered to minipigs beginning ten days before ligation of coronary artery and continued for 60 days surgery. Challenge with ep
probucol-treated minipigs. CLINICAL STUDIES: In a multicenter, randomized, double-blind study, the LRC-CPPT, 's hypercholesterolemic pa
treated with an oral bile acid sequestrant (cholestyramine) and a cholesteroillowering diet experienced average total and
cholesterol reductions greater than those obtained in the placebo group treated with diet alone. The cumulative seven incidence of the primary end point-combined incidence of definite $\mathrm{CHD}$ death and or definite nonfatal myocardial infarc was $7 \%$ in the chiolestyramine group and $8.6 \%$ in the placebo group. This was a $19 \%$ reduction in risk ( $P$ less than 0.05 ,
tail test) of the primary end point reflecting a $24 \%$ reduction in definite CHD death and a $19 \%$ reduction in nontatal my infarction. The subjects included in the study were middle-aged men ( $35-59$ years old) with serum cholesterol levels at least $265 \mathrm{mg} / \mathrm{d}$
no previous history of heart disease. It is not clear to what extent these findings can be extrapolated to other segments

The bile acid sequestrant, cholestyramine, was used in the above trial. Caution should be exercised in extrapolating these $r$ e to Lorelco since it differs from cholestyramine with regard to its mode of action, spectrum of cholesterol-lowering pote REFERENCES

. Consensus Development Panel. Lowering blood cholesterol to prevent heart disease. JAMA. 1985; 253:2080-2086 2. Fredrickson DS, Levy RI, Lees RS. Fat transport in lipoproteins - an integrated approach to mechanisms and disord
Engl J Med 1967:2763:34-44. 3. The Lipid Research Clinics Program. The Lipid Research Clinics coronary primary prevention trial results: I. Reduct Product Information as of June, 1988

MERRELL DOW PHARMACEUTICALS INC
Subsidiary of The DOW Chemical Company

vererit ow 